\title{
MCP-1 deficiency enhances browning of adipose tissue via increased M2 polarization
}

\author{
Monisha Rajasekaran 1,*, Ok-Joo Sul1,*, Eun-Kyung Choi ${ }^{1, \dagger}$, Ji-Eun Kim¹, Jae-Hee Suh ${ }^{2}$ and Hye-Seon Choi ${ }^{1}$ \\ 1Department of Biological Sciences, University of Ulsan, Ulsan, Korea \\ 2Department of Pathology, Ulsan University Hospital, Ulsan, Korea \\ Correspondence should be addressed to H-S Choi: hschoi@mail.ulsan.ac.kr \\ *(M Rajasekaran and O-J Sul contributed equally to this work) \\ ${ }^{\dagger}(\mathrm{E}-\mathrm{K}$ Choi is now at Department of Molecular \& Integrative Physiology, University of Michigan, Ann Arbor, Michigan, USA)
}

\begin{abstract}
Obesity is strongly associated with chronic inflammation for which adipose tissue macrophages play a critical role. The objective of this study is to identify monocyte chemoattractant protein-1 (MCP-1, CCL2) as a key player governing M1-M2 macrophage polarization and energy balance. We evaluated body weight, fat mass, adipocyte size and energy expenditure as well as core body temperature of $\mathrm{Ccl} 2$ knockout mice compared with wild-type mice. Adipose tissues, differentiated adipocyte and bone marrow-derived macrophages were assessed by qPCR, Western blot analysis and histochemistry. MCP-1 deficiency augmented energy expenditure by promoting browning in white adipose tissue and brown adipose tissue activity via increasing the expressions of Ucp1, Prdm16, Tnfrsf9, Ppargc1a, Nrf1 and Th and mitochondrial DNA copy number. MCP-1 abrogation promoted M2 polarization which is characterized by increased expression of Arg1, Chil3, II10 and KIf4 whereas it decreased M1 polarization by decreased p65 nuclear translocation and attenuated expression of Itgax, Tnf and Nos2, leading to increased browning of adipocytes. Enhanced M2 polarization and attenuated M1 polarization in the absence of MCP-1 are independent. Collectively, our results suggest that the action of MCP-1 in macrophages modulates energy expenditure by impairing browning in adipose tissue.
\end{abstract} Key Words - MCP-1

- M2 polarization

- browning

- adipose tissue

\section{Introduction}

Monocyte chemoattractant protein-1 (MCP-1, CCL2) acts as a chemoattractant for monocytes and memory $\mathrm{T}$ cells via the binding of its receptor, CC-chemokine receptor-2 (CCR2). CCR2 belongs to the G-protein-coupled seventransmembrane receptor superfamily. Studies on CCR2 and $\mathrm{Ccl} 2$ knockout (KO) mice have demonstrated that MCP-1 plays a critical role in monocyte chemotaxis and chronic inflammation (Dawson et al. 2003, Weisberg et al. 2006), suggesting that the MCP-1/CCR2 axis is a potential therapeutic target for inflammatory diseases such as rheumatoid arthritis, multiple sclerosis and obesityassociated metabolic syndrome.

Brown adipose tissue (BAT) catabolizes lipids to generate heat by expressing uncoupling protein 1 (UCP-1) rather than producing ATP. A lean and healthy phenotype is associated with elevated BAT in animals, suggesting that modulating BAT could be used for protection against obesity. BAT is present at distinct anatomical sites, whereas brown fat cells are also present in white adipose tissues (WAT) mainly at the subcutaneous depots, 
known as beige cells. Increased browning elevates energy expenditure and improves insulin sensitivity (Stanford et al. 2013). Browning has been shown to be promoted through several stimuli such as cold exposure, endurance exercise, calorie restriction, microbiota depletion and genetic manipulation (Guerra et al. 1998, Himms-Hagen et al. 2000, Fabbiano et al. 2016, Matesanz et al. 2017). Some stimuli act in an adipocyte-autonomous way, whereas others are mediated by macrophage polarization.

Macrophages can obtain distinct functional phenotypes, M1 and M2, via different polarization responses to environmental stimuli. M1 phenotypes are stimulated by proinflammatory cytokines or microbial products with high antigen presentation, inducible NOS (iNOS), TNF- $\alpha$ and CD11c (Komohara et al. 2016). Alternatively, IL-4-induced macrophage activation, M2, is different from classical M1 macrophage activation (Gordon \& Martinez 2010). M2 macrophages are characterized by increased CD206, arginase 1, Ym1 and IL-10. Macrophage polarization is modulated by various inflammatory modulators, signal molecules and transcription factors. Since it is highly dynamic, the phenotypes of polarized macrophages can be converted under physiological and pathological conditions, although the molecular mechanisms underlying phenotype switching remain to be elucidated.

It is generally accepted that obesity-related metabolic disease is highly associated with adipose tissue macrophages (ATMs) (Weisberg et al. 2003). Increased numbers of macrophages in adipose tissue are caused by macrophage infiltration and proliferation mediated by proinflammatory cytokines or saturated fatty acids that are elevated by obesity. Under physiological conditions, the phenotype of ATM is mainly M2, but M1 macrophage activation of ATM has been associated with insulin resistance in humans and in the mouse model (Weisberg et al. 2003), suggesting that M1 macrophage polarization is important in the pathogenesis of obesity and insulin resistance via maintenance of inflammatory conditions. Accumulating evidence indicates that M2 macrophages can improve insulin sensitivity (Olefsky \& Glass 2010, Choi et al. 2015). An increase in CD206, an M2 surface marker, has been reported as associated with protection from insulin resistance and remodeling of adipose tissue (Aron-Wisnewsky et al. 2009, Choi et al. 2015). Stimulation by IL-4, which acts as an M2-inducing cytokine, results in phosphorylation of STAT6 and facilitates nuclear translocation and target gene activation of $\mathrm{M} 2$ polarization (Pauleau et al. 2004). Although the precise molecular mechanisms driving M2 polarization are not completely understood, critical roles for nuclear receptors and specific coactivators such as PPAR $\gamma$, PPAR $\delta$ and PGC- $1 \alpha$ have been reported as regulators of the M2 phenotype (Charo 2007). Kruppel-like factors (KLFs) belong to a subfamily of a zinc finger class of DNA-binding transcriptional regulators and have also been identified as essential regulators of macrophage M1/M2 polarization (Liao et al. 2011).

Our studies have demonstrated that MCP-1 deficiency contributes to $\mathrm{M} 2$ macrophage polarization and increased energy expenditure by enhancing WAT browning and BAT activity.

\section{Materials and methods}

\section{Reagents and antibodies}

Recombinant M-CSF mice were obtained from R\&D Systems, Inc. BAY 11-7082 and JSH23 were obtained from Calbiochem. Lipofectamine 3000 and Lipofectamine ${ }^{\mathrm{TM}}$ RNAiMAX were purchased from Invitrogen. HRPconjugated secondary antibodies, antibodies against $\beta$-actin, p65, Lamin B1, control IgGs (mouse and rabbit), small interfering RNA (siRNA) against KLF4 (sc-41050) and scrambled siRNA (scRNA, sc-37007) were all obtained from Santa Cruz Biotechnology. Antibodies against UCP-1 and KLF4 were obtained from Novus Biologicals (Cambridge, UK) and $\beta$-actin (A5441) was purchased from Sigma. M-MLV reverse transcriptase and SYBR Green RealTime PCR Master Mixes were purchased from Promega. QIAzol reagent was obtained from Qiagen.

\section{Animals}

Ccl2 knockout (KO) mice in a C57BL/6J genetic background were purchased from the Jackson Laboratory and were provided by the University of Ulsan, Immunomodulation Research Center (IRC). The offspring genotypes were determined by Southern blot analysis of DNA from tail biopsies. All mice were housed in a pathogen-free animal facility of the IRC, and were handled in accordance with the guidelines of the Institutional Animal Care and Use Committee (IACUC) of the IRC, University of Ulsan. All animal procedures were approved by the IACUC of the IRC. The approval ID for this study is HSC-2014-020. Food intake and body weight were monitored daily and weekly, respectively. Oxygen consumption $\left(\mathrm{VO}_{2}\right)$ and carbon dioxide production $\left(\mathrm{VCO}_{2}\right)$ were measured for each mouse for $1 \mathrm{~m}$ every $5 \mathrm{~m}$ by using an indirect calorimetric system (Promethion; Sable Systems, Las Vegas, NV, USA) operated 
under constant environmental temperature $\left(23^{\circ} \mathrm{C}\right)$ and a 12 h light (07:00-19:00 h), 12 h dark (19:00-07:00 h) cycle. Mice in each chamber had free access to food and water. Energy expenditure was calculated using the Weir equation: $\mathrm{kcal} / \mathrm{h}=60 \times\left(0.003941 \times \mathrm{VO}_{2}+0.001106 \times \mathrm{VCO}_{2}\right)$. The raw data were normalized by body weight. Abdominal temperature was measured in mice using biotelemetry transmitters (Mini-Mitter, Bend, OR, USA) implanted into the abdominal cavity one week prior to the experiment. Prior to surgery, mice were anesthetized with tribromoethanol (250 mg/kg B.W., Sigma Aldrich). The output (frequency in $\mathrm{Hz}$ ) was monitored by a receiver (model RA 1000; Mini-Mitter) placed under each cage. A data acquisition system (Vital View; Mini-Mitter) was used for automatic control of data collection and analysis. Body temperature was recorded at $10 \mathrm{~m}$ intervals. Female $\mathrm{Ccl} 2^{+/+}$ (wild type (WT)) and Ccl2 KO mice that were 21-22 weeks old were killed by $\mathrm{CO}_{2}$ asphyxiation and cervical dislocation. Blood was taken through cardiac puncture, and tissues were immediately harvested. To determine adipocyte size, adipose tissues were fixed in 10\% formalin solution for $24 \mathrm{~h}$, embedded in paraffin, sectioned at $5 \mu \mathrm{m}$, and stained with hematoxylin-eosin (H\&E). Images were obtained with an Axio-Star Plus microscope (Carl Zeiss, Gottingen, Germany) and further analyzed with Image J software.

\section{Preparation of bone marrow-derived macrophages (BMMs)}

Femora and tibiae were removed aseptically and dissected free of adherent soft tissue. The bone ends were cut, and the marrow cavity was flushed with $\alpha$-MEM from one end of the bone using a sterile 21-gauge needle. The bone marrow was further agitated using a Pasteur pipette in order to obtain a single-cell suspension, which was washed twice and incubated on plates with M-CSF (20 ng/mL) (R\&D Systems) for $16 \mathrm{~h}$. Non-adherent cells were then harvested, layered on a Ficoll-Hypaque gradient and cultured for two more days. After two days, large populations of adherent monocyte/macrophage-like cells had formed on the bottoms of the culture plates, as previously described (Choi et al. 2018). The few nonadherent cells were removed by washing the dishes with phosphate-buffered saline (PBS), while adherent cells (BMM) were harvested and seeded on plates. The adherent cells were confirmed negative for CD3 and CD45R and positive for CD115. The absence of contaminating stromal cells was confirmed by lack of cell growth in the absence of M-CSF. Additional medium with M-CSF was added and cells were incubated for an additional 6-7 days.

\section{Preparation of stromal vascular cells (SVC) and adipocytes}

Subcutaneous fat and visceral fat were obtained from the inguinal and preovarian adipose tissue depot, respectively. The tissue was weighed, rinsed three times in PBS and minced. Tissue suspensions were centrifuged at $500 \boldsymbol{g}$ for $5 \mathrm{~m}$ and treated with type 2 collagenase $(1 \mathrm{mg} / \mathrm{mL}$; Sigma Chemical) for $90 \mathrm{~m}$ at $37^{\circ} \mathrm{C}$ with shaking. Cell suspensions were filtered through a $100 \mu \mathrm{m}$ filter and centrifuged at $500 \boldsymbol{g}$ for $5 \mathrm{~m}$. SVC pellets were incubated with RBC lysis buffer (eBioscience, San Diego, CA, USA) for $5 \mathrm{~m}$, centrifuged at $300 \mathrm{~g}$ for $5 \mathrm{~m}$, and resuspended for further analysis. For adipocyte differentiation, the SVCs were washed with PBS and resuspended in DMEM supplemented with 10\% FBS. The medium was changed every 2-3 days. When the cells were 80-90\% confluent, preadipocytes were induced to differentiate into adipocytes by incubating in culture medium supplemented with isobutylmethylxanthine $(0.5 \mathrm{mM})$, rosiglitazone $(1 \mu \mathrm{M})$, dexamethasone $(5 \mu \mathrm{M})$, T3 $(1 \mathrm{nM})$, and insulin $(0.5 \mu \mathrm{g} / \mathrm{mL})$ for two days, and then incubated with rosiglitazone $(1 \mu \mathrm{M})$, T3 $(1 \mathrm{nM})$, and insulin $(0.5 \mu \mathrm{g} / \mathrm{mL})$ for five more days in order to obtain fully differentiated adipocytes.

\section{siRNA transfections}

BMMs were transfected with small interfering RNA (siRNA) against Klf4 (siKLF4) or scrambled siRNA (scRNA) using Lipofectamine $^{\mathrm{TM}}$ RNAiMAX. Lipofectamine ${ }^{\mathrm{TM}}$ RNAiMAX $(2 \mu \mathrm{L})$ was diluted in $\alpha$-MEM $(50 \mu \mathrm{L})$ without serum. Then, an equal volume of $\alpha$-MEM containing $50 \mathrm{nM}$ of siRNA was added, and the mixture was incubated for $20 \mathrm{~min}$. The medium was removed, and fresh medium without serum was added. The resulting RNAiMAX/siRNA was added directly to cells, giving a final volume of $700 \mu \mathrm{L}$. After an $8 \mathrm{~h}$ incubation, the cells were refreshed with serumcontaining medium and cultured for the indicated times.

\section{RNA isolation and quantitative polymerase chain reaction ( $q P C R)$}

Total RNA was extracted from cells with QIAzol reagent and first strand cDNA was reverse-transcribed with random primers and M-MLV reverse transcriptase. Inhibition of reverse-transcription activity or PCR was https://joe.bioscientifica.com

https://doi.org/10.1530/JOE-19-0190
(C) 2019 Society for Endocrinology Published by Bioscientifica Ltd. Printed in Great Britain 
determined by sample dilution. qPCR was carried out using SYBR Green Real-Time PCR Master Mixes with the appropriate primers in MicroAmp Fast Reaction Tubes (8 tubes/strip) (Applied Biosystems, Foster City, CA, USA) on a StepOnePlus ${ }^{\mathrm{TM}}$ Real Time System. The specificity of each primer pair was confirmed by melting curve analysis and agarose-gel electrophoresis. The housekeeping Rps18 (18S rRNA) gene was amplified in parallel with the genes of interest. The relative amount of transcript was calculated based on the $2^{-\Delta \Delta C t}$ method (Livak \& Schmittgen 2001), where the $\Delta \Delta \mathrm{Ct}$ is the difference between the selected $\Delta \mathrm{Ct}$ value of a particular sample and the $\Delta \mathrm{Ct}$ of control. The mean expression of the target genes in WT was assigned an expression value of 1.0. The following primer sequences were used: $5^{\prime}$-ctggatagcctttcttctgctg-3' and 5'-gcacactgtgtccgaactc-3' (Itgax); 5'-aacggagaacgttggatttg-3' and $\quad 5^{\prime}$-cagcacaaggggttttcttc-3' (Nos2); 5'-ccagaccc tcactagatca-3' and $5^{\prime}$-cacttggtggtttgctacgac-3' (Tnf); 5'-cgggaagggagaagacact-3' and 5'-gagttcctcacgccaacg-3' (Klf4); 5'-tctgggtacaagatccetgaa-3' and $5^{\prime}$-tttctcc agtgtagccatcctt-3' (chitinase-like 3, Chil3); 5'-gctcttact gactggcatgag- $3^{\prime}$ and $5^{\prime}$-cgcagctctaggagcatgtg (Il10); $5^{\prime}$-ctcc aagccaaagtccttaga-3' and 5'-aggagctgtcattagggacatc-3' (Arg1); 5'-ccacgttggatgagtacacg-3' and 5'-ctgagcc tgggtcattttgt-3' (nuclear respiratory factor1, Nrf1); $5^{\prime}$-caa gtcagctgatgggtatgg- $3^{\prime}$ and $5^{\prime}$-tttccetgagccgaatcatcc- $3^{\prime}$ (transcription factor A, mitochondria, Tfam); $5^{\prime}$-ccetgtggtgagcttctctc-3' and $5^{\prime}$-gctcatagcctcctcctcct-3' (Tnfrsf9); $5^{\prime}$-actgccacacctccagtcatt- $3^{\prime}$ and $5^{\prime}$-ctttgcctcactcag gattgg-3' (Ucp 1); 5'-agccgtgaccactgacaacgag-3' and 5'-gctgcatggttctgagtgctaag-3' (Ppargc1a); 5'-cagcac ggtgaagccattc-3' and 5'-gcgtgcatccgcttgtg-3' (Prdm16); 5'-aagatcaaacctaccagccg-3' and 5'-tacgggtcaaacttcacagag-3' (Th); and 5'-atcagagagttgaccgcagttg-3' and $5^{\prime}$-aatgaacc gaagcacaccatag-3' (Rps18).

\section{Mitochondrial DNA}

Total DNA was extracted using the AccuPrep Genomic DNA Extraction Kit (Bioneer, Daejeon, Korea). mtDNA copy number was measured with real-time quantitative PCR. The primers $5^{\prime}$-ccacttcatcttaccattta- $3^{\prime}$ and 5'-atcgcatctgagtttaatc-3' (mouse cytochrome b, Mt-cytb) were used for mtDNA. The primers 5 '-gggagcctgagaaa cggc-3' and 5'-gggtcgggagtgggtaattt-3' (Rps18) were used for nuclear DNA (nDNA). Relative amounts of mtDNA and nDNA copy numbers were compared.

\section{Fractionation and Western blot analysis}

Cultured cells were harvested after washing with ice-cold PBS and lysed in extraction buffer $(50 \mathrm{mM}$ Tris- $\mathrm{HCl} \mathrm{pH}$ 8.0, $150 \mathrm{mM} \mathrm{NaCl}, 1 \mathrm{mM}$ EDTA, $0.5 \%$ Nonidet P-40 and $0.01 \%$ protease inhibitor mixture). Cells were fractionated using nuclear and cytoplasmic extraction reagents (Pierce) for detection of p65. Nuclear extracts were subjected to SDS-PAGE and transferred onto nitrocellulose. Membranes were blocked for $1 \mathrm{~h}$ with skim milk in Tris-buffered saline containing $0.1 \%$ Tween $20 \%$ and incubated overnight at $4^{\circ} \mathrm{C}$ with anti-p65 and anti-Lamin B1 (Santa Cruz). Cultured cells were harvested after washing with ice-cold PBS and then lysed in extraction buffer $(50 \mathrm{mM}$ Tris- $\mathrm{HCl}$ pH 8.0, $150 \mathrm{mM} \mathrm{NaCl}, 1 \mathrm{mM}$ EDTA, $0.5 \%$ Nonidet P-40, and $0.01 \%$ protease inhibitor mixture). Cell extracts were subjected to SDS-PAGE and transferred onto nitrocellulose. Membranes were blocked for $1 \mathrm{~h}$ with skim milk in Trisbuffered saline containing $0.1 \%$ Tween $20 \%$ and incubated overnight at $4^{\circ} \mathrm{C}$ with antibodies against KLF4, UCP- 1 and $\beta$-actin. Membranes were washed, incubated for $1 \mathrm{~h}$ with HRP-conjugated secondary antibodies and developed using chemiluminescence substrates.

\section{Statistical analysis}

Values are expressed as means of triplicate experiments \pm s.D. Each series of experiments was repeated at least three times. Statistical analysis was performed using a Student's t-test when two groups were compared or by one-way ANOVA, followed by Bonferroni post-hoc tests, if multiple groups were compared. A $P$ value $<0.05$ was considered statistically significant.

\section{Results}

\section{MCP-1 deficiency increases energy expenditure}

Our previous studies demonstrated that MCP-1 deficiency improves insulin resistance and decreased body weight that was increased due to loss of ovarian function (Kim et al. 2013). In the present study, we focused on the effect of MCP-1 on increasing body weight as well as fat mass. MCP-1 deficiency alone did not change body weight significantly in mice up to 18 weeks old compared to wild-type mice. However, in older mice, body weight decrease was significant in the absence of MCP-1 (Fig. 1A). Along with body weight, the fat masses of gonadal and https://joe.bioscientifica.com https://doi.org/10.1530/JOE-19-0190 (c) 2019 Society for Endocrinology Published by Bioscientifica Ltd.
Printed in Great Britain 

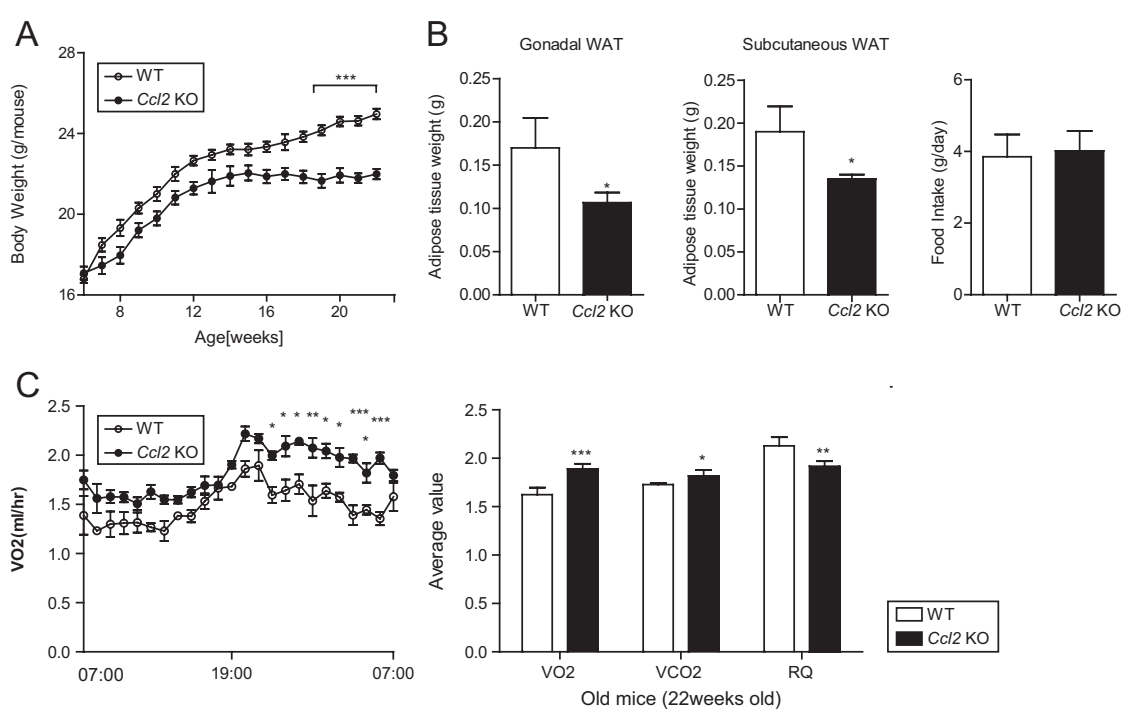

\section{Figure 1}

MCP-1 deficiency increases energy expenditure. (A) Body weight during the indicated time course for WT $(n=10)$ and $C c / 2 \mathrm{KO}(n=9)$ female mice under physiological conditions. (B) Fat mass in WT $(n=10)$ and $\mathrm{CCl} 2 \mathrm{KO}(n=9)$ mice at 21 weeks. (C)
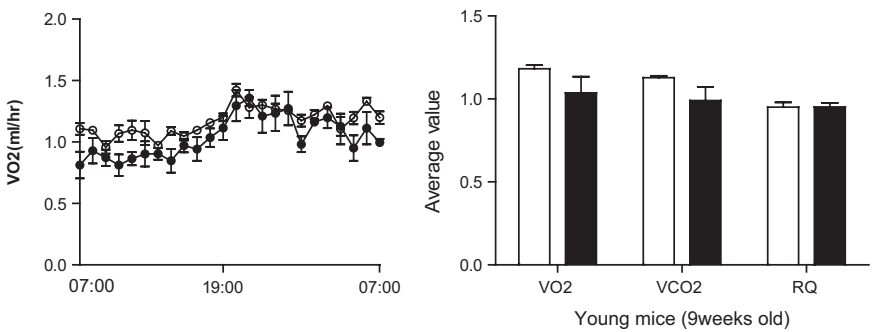
Energy balance between WT $(n=8)$ and $\mathrm{CCl} 2 \mathrm{KO}$ $(n=7)$ female mice. Mice at 22 weeks were examined in a metabolic cage over a 2-day period to measure energy expenditure (EE). EE was corrected for body weight. Food intake was measured daily. $* P<0.05 ; * \star P<0.01 ; * \star \star P<0.001$ compared with each corresponding control. Values are shown as the means of more than triplicate experiments \pm S.D. Similar results were obtained in three independent experiments.

subcutaneous WAT in mice at 22 weeks old were also markedly reduced in the absence of MCP-1 (Fig. 1B).

We next determined whether MCP-1 deficiency affected energy balance. Indirect calorimetric analysis demonstrated that MCP-1 deficiency at age of 22 weeksold female mice had higher $\mathrm{O}_{2}$ consumption than wild type (WT) in the absence of changes to daily food consumption during normal chow feeding (Fig. 1C). However, $\mathrm{O}_{2}$ consumption was not changed in the absence of MCP-1 with 9-weeks-old female mice (Fig. 1C). It is plausible that increased $\mathrm{O}_{2}$ consumption due to MCP-1 deficiency may be a major determinant of MCP-1-regulated body weight increase and led us to examine thermogenesis. When maintained at room temperature $\left(23^{\circ} \mathrm{C}\right)$, the core body temperature of $\mathrm{Ccl} 2 \mathrm{KO}$ mice exhibited a significant increase during the night period compared to core body temperature of WT mice (Fig. 1D), suggesting that loss of MCP-1 promotes energy expenditure via enhanced thermogenesis.

\section{MCP-1 deficiency promotes the development of browning in white adipose tissue and brown adipose tissue activity}

To determine whether decreased fat mass in the absence of MCP-1 was due to a difference in adipocyte number and area, each were calculated from histological examination of gonadal and subcutaneous fat. As shown in Fig. 2A, the absence of MCP-1 increased the number of adipocytes, whereas it decreased the area of adipocytes significantly for both gonadal and subcutaneous fat. These properties resulting from a deficiency in MCP-1 are characteristic of mature beige adipocytes. That prompted us to investigate whether MCP-1 deficiency affects the browning of WAT. We found that subcutaneous fat from $\mathrm{Ccl} 2 \mathrm{KO}$ mice exhibited a marked increase in browning markers, Ucp1, Ppargc1a (peroxisome proliferator-activated receptor $\gamma$ coactivator-1), Prdm16 (PR domain containing 16) and Tnfrsf9 (CD137) (Fig. 2B). Because beige adipocytes usually contain high levels of mitochondria, we also examined mitochondrial DNA copy number normalized to genomic DNA content. MCP-1 deficiency significantly increased mitochondrial copy number as well as the mitochondria biogenesis markers, Nrf1 and Tfam (Fig. 2B). The expression levels of tyrosine hydroxylase, a rate-limiting enzyme in the biosynthesis of catecholamines, which activate both brown and beige fat, were significantly elevated in subcutaneous WAT of Ccl2 KO mice (Fig. 2B). These results indicated that higher browning activity was concomitant with accelerated catecholamine synthesis in the absence of MCP-1. The increased browning of the subcutaneous fat depot was 
A
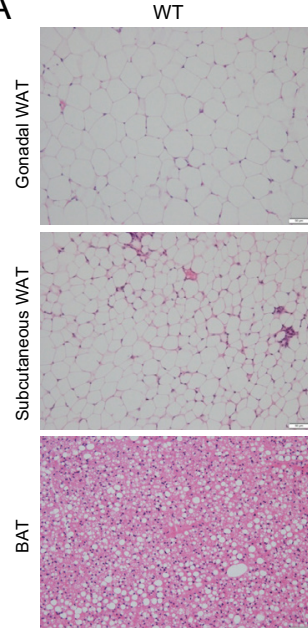

MCP-1 KO

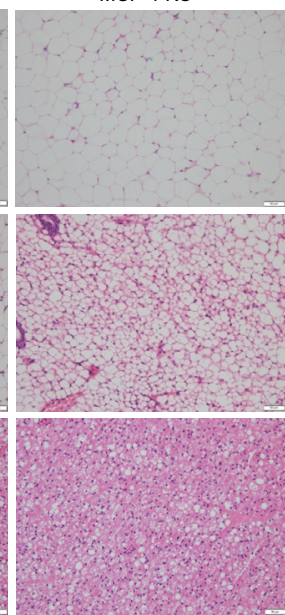

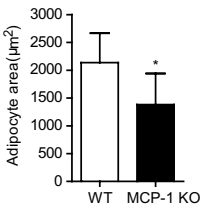

$\left.\widetilde{\widetilde{E}}_{800}^{1000}\right] \mathrm{T}$
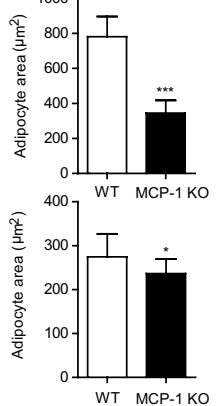
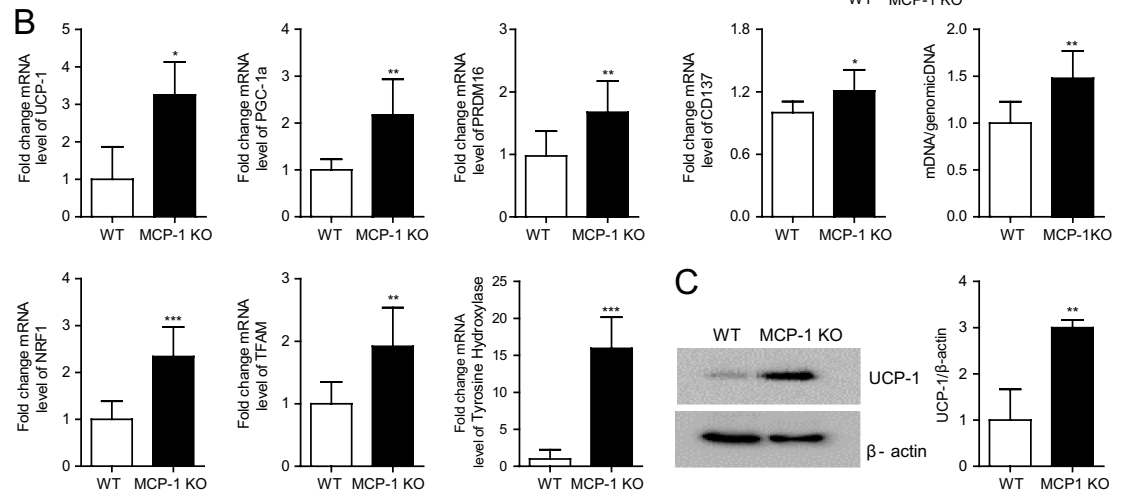

C
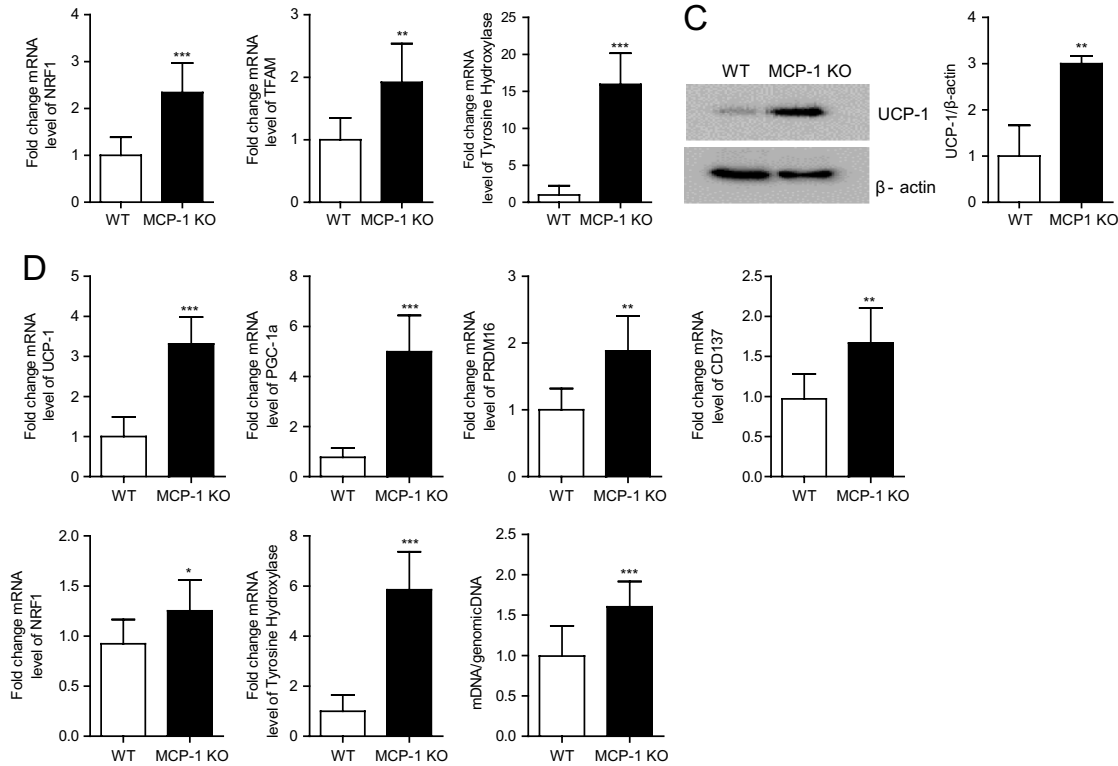

confirmed by Western blotting as shown by increased UCP-1 protein levels in subcutaneous fat of $\mathrm{Ccl} 2 \mathrm{KO}$ mice (Fig. 2C). We wondered whether MCP-1 deficiency also affected BAT. MCP-1 deficiency increased adipocyte number and decreased adipocyte size in BAT to a lesser degree than in gonadal and subcutaneous fat (bottom panel of Fig. 2A). MCP-1 deficiency augmented expression of key browning genes (Ucp1, Prdm16, Tnfrsf9, Ppargc1a, Nrf1 and Th) and mitochondrial DNA copy number in BAT (Fig. 2D). These results suggested that MCP-1 deficiency

\section{Figure 2}

MCP-1 deficiency promotes the development of browning in white adipose tissue and brown adipose tissue activity. (A) Adipocyte size in subcutaneous WAT, gonadal WAT and brown adipose tissue (BAT) from WT $(n=6)$ and $\mathrm{Cc} / 2 \mathrm{KO}$ $(n=4)$ mice at 21 weeks was measured from H\&E-stained sections using Image J software. Representative photos of adipocytes. Scale bar: $50 \mu \mathrm{m}$. (B and D) qPCR analysis of mitochondria biogenesis and browning-associated genes in subcutaneous WAT (B) and BAT (D). Data were normalized to the expression of Rps $18 \mathrm{mRNA}$ in each sample (WT; $n=6, C \mathrm{Cl} 2 \mathrm{KO} ; n=5$ ). (C) Tissue lysates from subcutaneous WAT were subjected to Western blot analysis using an anti-UCP-1 antibody. $\beta$-Actin was used as a loading control. $\star P<0.05 ; * \star P<0.01 ; * \star * P<0.001$ compared with each corresponding control. Values are shown as the means of more than triplicate

experiments \pm S.D. $(n=3 \sim 5)$ with at least three times of repetitive experiments. A full color version of this figure is available at https://doi. org/10.1530/JOE-19-0190. plays a role in enhanced BAT activity as well as browning in WAT.

\section{MCP-1 deficiency increases M2 polarization and decreases M1 polarization to induce WAT browning}

To assess whether WAT browning is an adipocyteautonomous phenotype in the absence of MCP-1, preadipocytes from $\mathrm{WT}$ and $\mathrm{Ccl} 2 \mathrm{KO}$ mice were differentiated into mature adipocytes and gene expression associated 
with browning and mitochondrial biogenesis was analyzed. As shown in Fig. 3A, there were no significant increases in Ucp1, Prdm16, Tnfrsf9, Ppargc1a, Nrf1 and Tfam in the subcutaneous WAT of Ccl2 KO mice. In adipose tissue, infiltrating monocytes are differentiated into macrophages with specialized functional phenotypes according to the local microenvironment. Recent findings have reported that M2 polarization induces WAT browning (Liu et al. 2015). This observation prompted us to examine whether MCP-1 deficiency may be coupled to browning through a shift in M1-M2 polarization of ATMs in a macrophage autonomous manner. As shown in Fig. 3B, BMMs from $\mathrm{Ccl} 2 \mathrm{KO}$ mice exhibited increased expression of Arg1, Chil3 and Il10 and decreased expression of Itgax, Tnf and Nos 2 compared to the expression levels in WT mice. Next, we assessed cross-talk between M2 polarized macrophages and beige adipocytes and, using conditioned medium from macrophages, monitored whether MCP-1 deficiency in macrophages may affect this cross-talk.
We used UCP-1-expressing beige adipocytes differentiated from preadipocytes and treated them with conditioned medium from BMMs. The conditioned medium of BMMs from $\mathrm{Ccl} 2 \mathrm{KO}$ mice significantly increased the expression of $U c p 1$ and other beige markers, including Ppargc1a, Nrf1 and Tnfrsf9, as compared to the expression in WT mice (Fig. 3C). Blockade of MCP-1 by anti-MCP-1 Ab in the conditioned medium of WT BMM also showed enhanced expressions of Ucp1, Ppargc1a, Nrf1 and Tnfrsf9 compared to mouse IgG. These results suggested that increased WAT browning was due to augmented M2 polarization and reduced M1 polarization of ATMs in the absence of MCP-1.

\section{Enhanced M2 polarization and attenuated M1 polarization in the absence of MCP-1 are independent}

To explore the mechanisms by which MCP-1 deficiency elevates M2 polarization, we examined the effects of
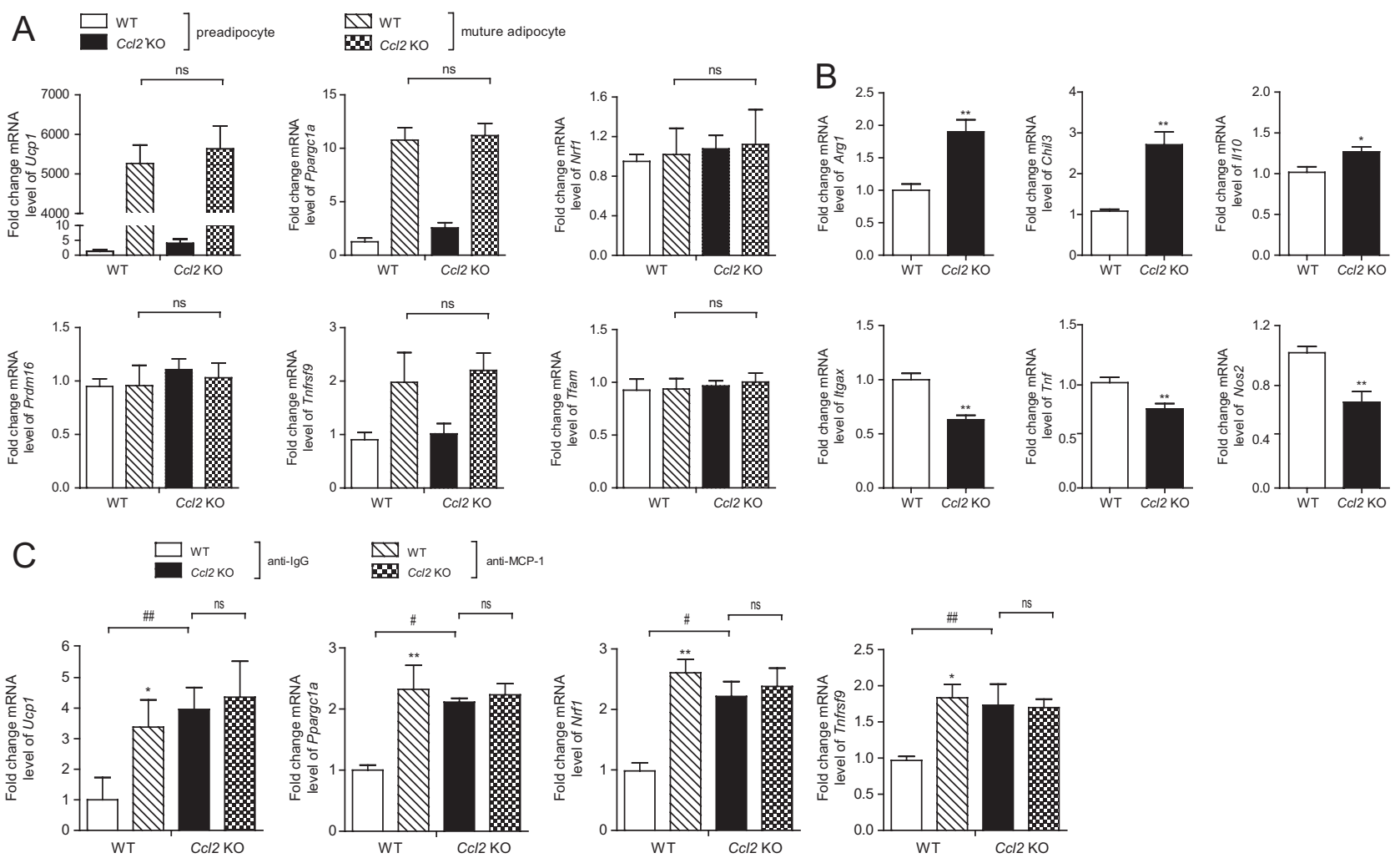

Figure 3

MCP-1 deficiency increases M2 polarization and decreases M1 polarization to induce WAT browning. (A) qPCR analysis of mitochondria biogenesis and browning-associated genes in in vitro differentiated adipocytes from subcutaneous WAT of WT and C Cl2 KO mice. (B) BMMs from WT and CCl2 KO mice were prepared and incubated with M-CSF (30 ng/mL) for 6 days. Total RNA was analyzed by qPCR to quantify the expression of Itgax, Tnf, Nos2, Arg1, Chil3 and //10. (C) qPCR analysis of mitochondria biogenesis and browning-associated genes in cells differentiated from primary preadipocytes for 7 days across the differentiation process with the conditioned medium using BMMs isolated from WT and $\mathrm{Ccl} 2 \mathrm{KO}$ mice. The conditioned medium was supplemented with a neutralizing anti-MCP-1 Ab or a nonspecific control IgG (mouse IgG). $* P<0.05$; $* \star P<0.01$; compared with each corresponding control. Values are shown as the means of more than triplicate experiments \pm S.D. $(n=3 \sim 5)$ with at least three times of repetitive experiments. 
MCP-1 deficiency on the expression of Klf4 (Kruppel-like factor 4), which has been identified as a critical regulator of M2 macrophage polarization (Liao et al. 2011). As shown in Fig. 4A, MCP-1 deficiency increased Klf4 mRNA and protein levels in macrophages. In addition, the expression of Klf4 mRNA and protein levels were also increased in AT from $\mathrm{Ccl} 2 \mathrm{KO}$ mice compared to Klf4 mRNA and protein levels in WT mice (Fig. 4B). Next, to confirm the role of KLF4 in the absence of MCP-1, we used siRNA to determine the effect of KLF4 down-regulation on macrophage polarization. Knock-down of endogenous Klf4 resulted in a dramatic decrease of M2-specific genes, Arg1, Chil3 and IllO in BMMs from Ccl2 $\mathrm{KO}$ mice. This reduction was less in WT mice (Fig. 4C). However, there were no significant changes in M1-specific genes, Itgax, Tnf and Nos2, in BMMs from both mice genotypes (Fig. 4C). These results suggested that MCP-1 deficiency-induced KLF4 plays a critical role in M2 polarization, but it does not affect the decrease in M1 polarization. Since our previous data had shown that MCP-1 deficiency reduced NF-kB-DNA binding in BMM upon RANKL stimulation (Sul et al. 2012), we evaluated the nuclear transport of p65 in BMMs upon MCP-1 stimulation. As shown in Fig. 4D, in BMMs from WT mice, p65 was detectable after $1 \mathrm{~h}$ and maintained for up to $6 \mathrm{~h}$ with MCP-1 stimulation; however, these levels were reduced in BMMs from Ccl2 $\mathrm{KO}$ mice. This result prompted us to determine whether decreased M1 polarization induced by MCP-1 deficiency contributed to elevation of M2 polarization. The expression of KLF4 in the presence or absence of MCP-1 was examined with pharmacological inhibitors of NF-KB activation, BAY11-7082 and JSH23. Addition of BAY11-7082 and JSH23 decreased expression of Itgax, Tnf and Nos2 in BMMs from WT mice (Fig. 4E). However, inhibition did not
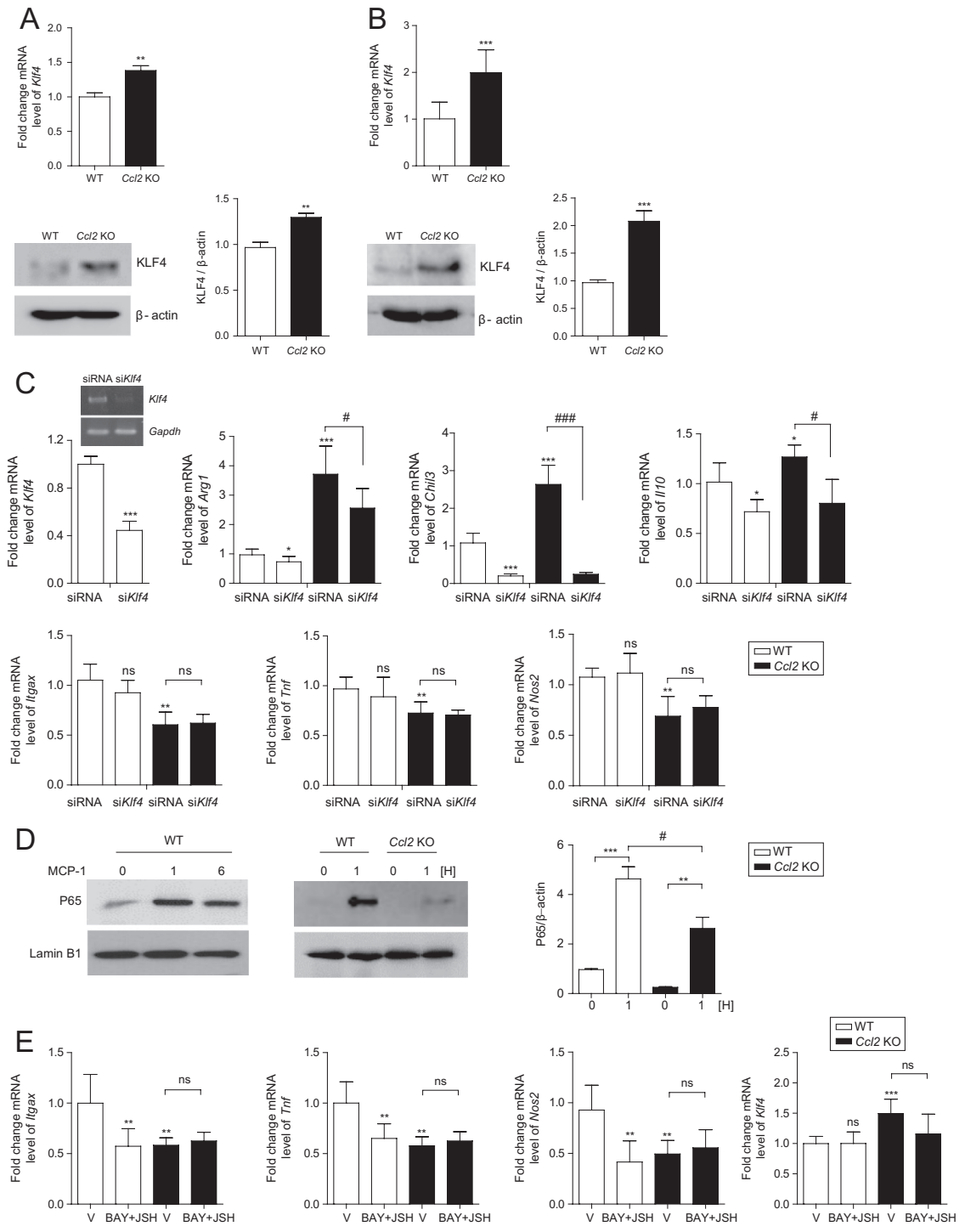

\section{Figure 4}

Enhanced M2 polarization and attenuated M1 polarization in the absence of MCP-1 are independent. ( $A$ and $B$ ) BMMs from WT and $\mathrm{CCl} 2$ $\mathrm{KO}$ mice were prepared and incubated with M-CSF (30 ng/mL) for 6 days (A), and subcutaneous WAT from WT and $\mathrm{CC} / 2 \mathrm{KO}$ mice were prepared (B). RNAs were analyzed by qPCR to quantify the expression of $K I f 4$, and cell lysates were subjected to Western blot analysis using an anti-KLF4 antibody. $\beta$-Actin was used as a loading control. (C) BMMs from WT and $\mathrm{Ccl} 2 \mathrm{KO}$ mice were incubated with M-CSF (30 ng/mL) for 6 days and then washed thoroughly. Cells were transfected with $50 \mathrm{nM}$ of siKIf4 or ScRNA, and incubated for an additional 2 days with M-CSF. Silencing of KLF4 using siRNA was confirmed by RT-PCR and qPCR. Total RNA was analyzed by qPCR to quantify the expression of Arg1, Chil3, 1110, Itgax, Tnf and Nos2. (D) BMMs from WT and $\mathrm{Ccl} 2 \mathrm{KO}$ mice were incubated with $\mathrm{M}$-CSF (30 ng/mL) for 6 days, and stimulated with MCP-1 for the indicated time. Nuclear fractions were harvested from cultured cells and subjected to Western blot analysis using an anti-p65 antibody. A lamin B1 antibody was used for normalization of nuclear extracts. (E) BMMs from WT and $\mathrm{Cc} / 2$ $\mathrm{KO}$ mice were incubated with $\mathrm{M}-\mathrm{CSF}(30 \mathrm{ng} / \mathrm{mL})$ for 6 days and then incubated for 2 days in the presence or absence of BAY 11-7032 $(5 \mu \mathrm{M})$ and JSH-23 $(10 \mu \mathrm{M})$. RNAs were analyzed by qPCR to quantify the expression of Klf4, Itgax, Tnf and Nos2. $* P<0.05 ; * * P<0.01 ; * * * P<0.001$ compared with each corresponding control. $\# P<0.05$; \#\#\# $P<0.001$ between the indicated pair. Values are shown as the means of more than triplicate experiments \pm S.D. $(n=3 \sim 5)$ with at least three times of repetitive experiments. https://joe.bioscientifica.com https://doi.org/10.1530/JOE-19-0190 (c) 2019 Society for Endocrinology Published by Bioscientifica Ltd. Printed in Great Britain 
affect the increased expression of Klf4 in the absence of MCP-1 (Fig. 4E). These results suggested that augmented M2 polarization and reduced M1 polarization in the absence of MCP-1 were independent.

\section{Discussion}

Recent studies have demonstrated that immune cells are associated with pathophysiology occurring in AT. Especially critical role of M1 macrophages in AT has been reported to result in insulin resistance involved in obesity due to diet (Romeo et al. 2012, Giralt \& Villarroya 2013) or hormonal changes (Kim et al. 2013). Various immune cells including mainly M2 macrophages with eosinophils, ILC2 cells and T lymphocytes also affect WAT browning and BAT activities (Villarroya et al. 2018).

M2 macrophages have been demonstrated to be recruited to WAT and BAT after cold exposure (Qiu et al. 2014) or multiple other stimuli such as caloric restriction (Fabbiano et al. 2016), microbiota depletion or genetic manipulations (Shan et al. 2017). Injection of engineered anti-inflammatory macrophages also facilitates WAT browning (Liu et al. 2015). However, it is not clearly defined how M2 macrophage recruitment is associated with thermogenic activation in BAT and WAT browning. M2 macrophages have been considered to express tyrosine hydroxylase that is a rate-limiting enzyme for biosynthesis of norepinephrine (Daubner et al. 2011). On the contrary, recent studies by Fischer et al. (2017) have raised question whether M2 produces catecholamine. They showed that M2 induction did not change catecholamine level in vitro and in vivo. Lee et al. (2016) have reported that increased browning by $\mathrm{M} 2$ is due to efficient removal of cell remnant during tissue remodeling related to WAT browning. Cereijo et al. (2018) have demonstrated that macrophage recruitment and M2 polarization in AT contribute to browning through direct M2 macrophage recruitment using brown-adipocyte-derived CXCL14. Further studies are required to clarify these controversial issues.

We have demonstrated that MCP-1 deficiency promotes browning in WAT as well as BAT activity in aged female mice. Our previous data showed that the absence of MCP-1 improved insulin resistance and decreased fat mass upon loss of ovarian function. Moreover, WAT and BAT in mice lacking MCP-1 have elevated expression of Ucp1, a marker of browning, other important browning factors (Ppargc1a, Prdm16 and Tnfrsf9), and Th that is required for norepinephrine. In addition, MCP-1 deficiency increased total mitochondria content along with other mitochondria biogenesis markers, specifically $\mathrm{Nrf1}$ and Tfam. Our data showed the physiological relevance of this effect in regulating whole-body metabolism and weight control. A lack of MCP-1 significantly increased energy expenditure, controlled for body weight and increased body temperature.

Enhanced WAT browning in the absence of MCP-1 was not adipocyte-autonomous since no changes in expression of browning-related genes were found between differentiated adipocytes from WT and $\mathrm{Ccl} 2 \mathrm{KO}$ mice. However, when conditioned media from BMMs of $\mathrm{Ccl} 2 \mathrm{KO}$ mice was added to preadipocytes during differentiation, adipocytes exhibited higher expression levels of the browning markers Ucp1, Ppargc1a and Tnfrsf9 versus when BMM-conditioned media from WT mice was used. Thus, there may be a role for ATM on WAT browning in the absence of MCP-1. The role of MCP-1 deficiency in modulating browning was strengthened by
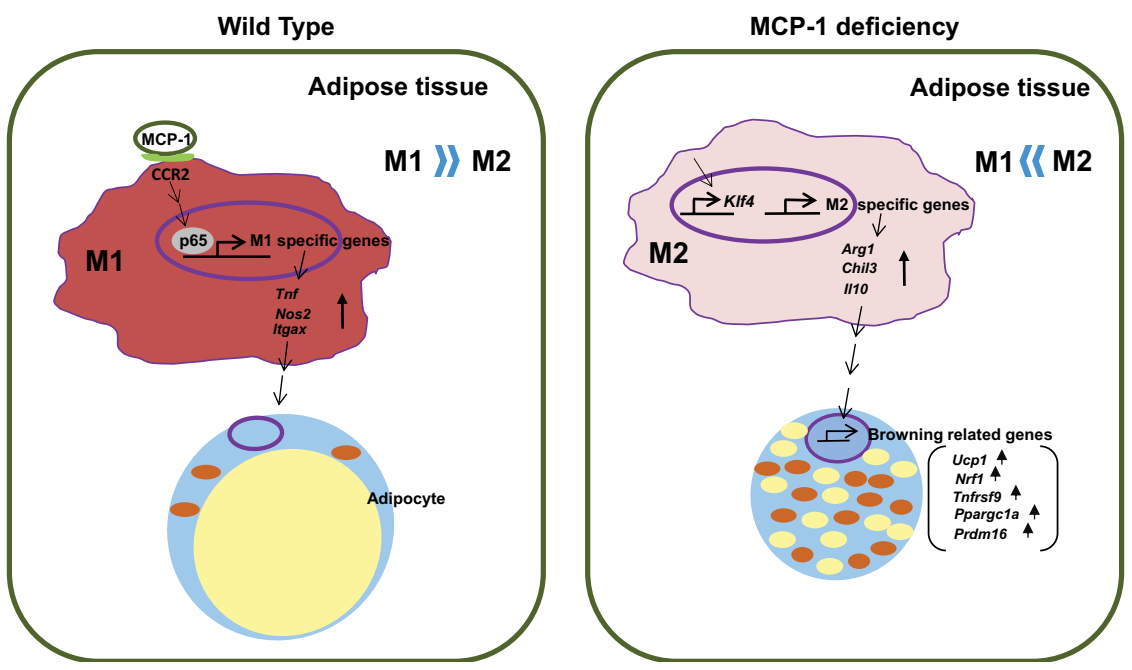

Figure 5

MCP-1 deficiency promotes M2, while it decreases M1 polarization of macrophages, leading to increased browning of adipocytes in adipose tissue. A full color version of this figure is available at https://doi.org/10.1530/JOE-19-0190. 
the finding that BMMs from $\mathrm{Ccl} 2 \mathrm{KO}$ mice enhanced M2 polarization as observed through elevated expression of Arg1, Chil3, Il10 and Klf4 and decreased M1 polarization as observed through reduced levels of Itgax, Tnf and Nos2 (Fig. 5). Although it is controversial, the positive effect of M2 macrophages on browning has been proposed to be to release norepinephrine via up-regulating tyrosine hydroxylase (Villarroya et al. 2018). Our data also demonstrated that the transcript of tyrosine hydroxylase in the absence of MCP-1 was increased in adipose tissue, but not in BMM. However, it is plausible that M2 macrophages in MCP-1 deficiency could release other factors to increase browning. Further studies need to be performed.

UCP-1 plays a critical role in regulating energy balance and thermogenesis (Nedergaard et al. 2001). The expression of UCP-1 is controlled partly by norepinephrine (Ramseyer \& Granneman 2016), which has been reported to be produced by M2 macrophages (Qiu et al. 2014, Hui et al. 2015, Fabbiano et al. 2016). Increased M2 macrophage polarization was responsible for elevated browning in WAT. Our results also showed that MCP-1 deficiency elevated the expression of tyrosine hydroxylase, which is a rate-limiting enzyme in catecholamine biosynthesis and supports a role for MCP-1 deficiency in elevated UCP-1. Similar phenomena have been observed in several studies. Microbiota depletion and calorie restriction had increased WAT browning resulting from increased type 2 signaling and M2 macrophage polarization in the subcutaneous WAT (Fabbiano et al. 2016). Furthermore, it has been shown that genetic and pharmacological blockade of the LPS-TLR4 pathway elevates beige fat development (Fabbiano et al. 2016). It has also been demonstrated that administration of M2 macrophages derived from RIP140 knockout mice induces WAT browning (Liu et al. 2015), confirming the concept that delivery of anti-inflammatory macrophages can facilitate WAT browning.

We also demonstrated that in BMMs, MCP-1 deficiency increased KLF4, an important regulator of M2 macrophage polarization (Liao et al. 2011) as well as decreased NF-кB activation, as evaluated by p65 nuclear localization (Fig. 5). Down-regulation of Klf4 reduced Itgax, Tnf and Il10 significantly in the absence of MCP-1, but did not change Itgax, Tnf and Il10. Conversely, blockade of NF-кB activation using pharmacological inhibitors reduced the expression of Itgax, Tnf and Nos 2 without a change in Klf4 expression. These results suggested that elevated M2 polarization and attenuated M1 polarization due to MCP-1 deficiency were independent of each other.
Our finding established a role for MCP-1 deficiency in enhanced browning of AT through the regulation of body energy balance and M2 polarization, suggesting a potential therapy to reduce body weight.

\section{Declaration of interest}

The authors declare that there is no conflict of interest that could be perceived as prejudicing the impartiality of the research reported.

\section{Funding}

This work was supported by the Korea Healthcare Technology R\&D Project (HI18C0375) funded by the Ministry of Health and by the Basic Science Research Programs (2014R1A6A1030318) of the NRF.

\section{Author contribution statement}

The study was designed by M R, O J S and H S C, and was performed by $M R, O J S, E K C$, J E K and J H S. The manuscript was written by M R, O J S and HSC.

\section{References}

Aron-Wisnewsky J, Tordjman J, Poitou C, Darakhshan F, Hugol D, Basdevant A, Aissat A, Guerre-Millo M \& Clément K 2009 Human adipose tissue macrophages: $\mathrm{m} 1$ and $\mathrm{m} 2$ cell surface markers in subcutaneous and omental depots and after weight loss. Journal of Clinical Endocrinology and Metabolism 94 4619-4623. (https://doi. org/10.1210/jc.2009-0925)

Cereijo R, Gavaldà-Navarro A, Cairó M, Quesada-López T, Villarroya J, Morón-Ros S, Sánchez-Infantes D, Peyrou M, Iglesias R, Mampel T, et al. 2018 CXCL14, a brown adipokine that mediates brownfat-to-macrophage communication in thermogenic adaptation. Cell Metabolism 28 750.e6-763.e6. (https://doi.org/10.1016/j. cmet.2018.07.015)

Charo IF 2007 Macrophage polarization and insulin resistance: PPARgamma in control. Cell Metabolism 6 96-98. (https://doi. org/10.1016/j.cmet.2007.07.006)

Choi EK, Park HJ, Sul OJ, Rajasekaran M, Yu R \& Choi HS 2015 Carbon monoxide reverses adipose tissue inflammation and insulin resistance upon loss of ovarian function. American Journal of Physiology: Endocrinology and Metabolism 308 E621-E630. (https://doi. org/10.1152/ajpendo.00458.2014)

Choi EK, Rajasekaran M, Sul OJ, Joe Y, Chung HT, Yu R \& Choi HS 2018 Impaired insulin signaling upon loss of ovarian function is associated with a reduction of tristetraprolin and an increased stabilization of chemokine in adipose tissue. Molecular and Cellular Endocrinology 461 122-131. (https://doi.org/10.1016/j.mce.2017.09.002)

Daubner SC, Le T \& Wang S 2011 Tyrosine hydroxylase and regulation of dopamine synthesis. Archives of Biochemistry and Biophysics 1 1-12. (https://doi.org/10.1016/j.abb.2010.12.017)

Dawson J, Miltz W, Mir AK \& Wiessner C 2003 Targeting monocyte chemoattractant protein-1 signalling in disease. Expert Opinion on Therapeutic Targets 7 35-48. (https://doi.org/10.1517/14728222.7.1.35)

Fabbiano S, Suárez-Zamorano N, Rigo D, Veyrat-Durebex C, Stevanovic Dokic A, Colin DJ \& Trajkovski M 2016 Caloric restriction leads https://joe.bioscientifica.com https://doi.org/10.1530/JOE-19-0190 (c) 2019 Society for Endocrinology Published by Bioscientifica Ltd. Printed in Great Britain 
to browning of white adipose tissue through type 2 immune signaling. Cell Metabolism 24 434-446. (https://doi.org/10.1016/j. cmet.2016.07.023)

Fischer K, Ruiz HH, Jhun K, Finan B, Oberlin DJ, van der Heide V, Kalinovich AV, Petrovic N, Wolf Y, Clemmensen C, et al. 2017 Alternatively activated macrophages do not synthesize catecholamines or contribute to adipose tissue adaptive thermogenesis. Nature Medicine 23 623-630. (https://doi.org/10.1038/ nm.4316)

Giralt M \& Villarroya F 2013 White, brown, beige/Brite: different adipose cells for different functions? Endocrinology 154 2992-3000. (https:// doi.org/10.1210/en.2013-1403)

Gordon S \& Martinez FO 2010 Alternative activation of macrophages: mechanism and functions. Immunity 32 593-604. (https://doi. org/10.1016/j.immuni.2010.05.007)

Guerra C, Koza RA, Yamashita H, Walsh K \& Kozak LP 1998 Emergence of brown adipocytes in white fat in mice is under genetic control. Effects on body weight and adiposity. Journal of Clinical Investigation 102 412-420. (https://doi.org/10.1172/JCI3155)

Himms-Hagen J, Melnyk A, Zingaretti MC, Ceresi E, Barbatelli G \& Cinti S 2000 Multilocular fat cells in WAT of Cl-316243-treated rats derive directly from white adipocytes. American Journal of Physiology: Cell Physiology 279 C670-C681. (https://doi.org/10.1152/ ajpcell.2000.279.3.C670)

Hui X, Gu P, Zhang J, Nie T, Pan Y, Wu D, Feng T, Zhong C, Wang Y, Lam KS, et al. 2015 Adiponectin enhances cold-induced browning of subcutaneous adipose tissue via promoting M2 macrophage proliferation. Cell Metabolism 22 279-290. (https://doi.org/10.1016/j. cmet.2015.06.004)

Kim WK, Choi EK, Sul OJ, Park YK, Kim ES, Yu R, Suh JH \& Choi HS 2013 Monocyte chemoattractant protein-1 deficiency attenuates oxidative stress and protects against ovariectomy-induced chronic inflammation in mice. PLOS ONE 8 e72108. (https://doi.org/10.1371/ journal.pone.0072108)

Komohara Y, Fujiwara Y, Ohnishi K, Shiraishi D \& Takeya M 2016 Contribution of macrophage polarization to metabolic diseases. Journal of Atherosclerosis and Thrombosis 23 10-17. (https://doi. org $/ 10.5551 /$ jat.32359)

Lee YH, Kim SN, Kwon HJ, Maddipati KR \& Granneman JG 2016 Adipogenic role of alternatively activated macrophages in $\beta$-adrenergic remodeling of white adipose tissue. American Journal of Physiology: Regulatory, Integrative and Comparative Physiology 1 55-65. (https://doi.org/10.1152/ajpregu.00355.2015)

Liao X, Sharma N, Kapadia F, Zhou G, Lu Y, Hong H, Paruchuri K, Mahabeleshwar GH, Dalmas E, Venteclef N, et al. 2011 Krüppellike factor 4 regulates macrophage polarization. Journal of Clinical Investigation 121 2736-2749. (https://doi.org/10.1172/JCI45444)

Liu PS, Lin YW, Burton FH \& Wei LN 2015 Injecting engineered antiinflammatory macrophages therapeutically induces white adipose tissue browning and improves diet-induced insulin resistance. Adipocyte 4 123-128. (https://doi.org/10.4161/21623945.2014.981438)

Livak KJ \& Schmittgen TD 2001 Analysis of relative gene expression data using real-time quantitative PCR and the 2(-delta delta C(T) method. Methods 25 402-408. (https://doi.org/10.1006/meth.2001.1262)
Matesanz N, Bernardo E, Acín-Pérez R, Manieri E, Pérez-Sieira S, Hernández-Cosido L, Montalvo-Romeral V, Mora A, Rodríguez E, Leiva-Vega L, et al. 2017 MKK6 controls T3-mediated browning of white adipose tissue. Nature Communications 8 856. (https://doi. org/10.1038/s41467-017-00948-z)

Nedergaard J, Golozoubova V, Matthias A, Asadi A, Jacobsson A \& Cannon B 2001 UCP1: the only protein able to mediate adaptive non-shivering thermogenesis and metabolic inefficiency. Biochimica et Biophysica Acta 1504 82-106. (https://doi.org/10.1016/S00052728(00)00247-4)

Olefsky JM \& Glass CK 2010 Macrophages, inflammation, and insulin resistance. Annual Review of Physiology 72 219-246. (https://doi. org/10.1146/annurev-physiol-021909-135846)

Pauleau AL, Rutschman R, Lang R, Pernis A, Watowich SS \& Murray PJ 2004 Enhancer-mediated control of macrophage-specific arginase I expression. Journal of Immunology 172 7565-7573. (https://doi. org/10.4049/jimmunol.172.12.7565)

Qiu Y, Nguyen KD, Odegaard JI, Cui X, Tian X, Locksley RM, Palmiter RD \& Chawla A 2014 Eosinophils and type 2 cytokine signaling in macrophages orchestrate development of functional beige fat. Cell 157 1292-1308. (https://doi.org/10.1016/j.cell.2014.03.066)

Ramseyer VD \& Granneman JG 2016 Adrenergic regulation of cellular plasticity in brown, beige/Brite and white adipose tissues. Adipocyte $\mathbf{5}$ 119-129. (https://doi.org/10.1080/21623945.2016.1145846)

Romeo GR, Lee J \& Shoelson SE 2012 Metabolic syndrome, insulin resistance, and roles of inflammation - mechanisms and therapeutic targets. Arteriosclerosis, Thrombosis, and Vascular Biology 32 1771-1776. (https://doi.org/10.1161/ATVBAHA.111.241869)

Shan B, Wang X, Wu Y, Xu C, Xia Z, Dai J, Shao M, Zhao F, He S, Yang L, et al. 2017 The metabolic ER stress sensor IRE1 $\alpha$ suppresses alternative activation of macrophages and impairs energy expenditure in obesity. Nature Immunology 18 519-529. (https://doi.org/10.1038/ni.3709)

Stanford KI, Middelbeek RJ, Townsend KL, An D, Nygaard EB, Hitchcox KM, Markan KR, Nakano K, Hirshman MF, Tseng YH, et al. 2013 Brown adipose tissue regulates glucose homeostasis and insulin sensitivity. Journal of Clinical Investigation 123 215-223. (https://doi. org/10.1172/JCI62308)

Sul OJ, Ke K, Kim WK, Kim SH, Lee SC, Kim HJ, Kim SY, Suh JH \& Choi HS 2012 Absence of MCP-1 leads to elevated bone mass via impaired actin ring formation. Journal of Cellular Physiology 227 1619-1627. (https://doi.org/10.1002/jcp.22879)

Villarroya F, Cereijo R, Villarroya J, Gavaldà-Navarro A \& Giralt M 2018 Toward an understanding of how immune cells control brown and beige adipobiology. Cell Metabolism 27 954-961. (https://doi. org/10.1016/j.cmet.2018.04.006)

Weisberg SP, McCann D, Desai M, Rosenbaum M, Leibel RL \& Ferrante AW Jr 2003 Obesity is associated with macrophage accumulation in adipose tissue. Journal of Clinical Investigation 112 1796-1808. (https://doi.org/10.1172/JCI19246)

Weisberg SP, Hunter D, Huber R, Lemieux J, Slaymaker S, Vaddi K, Charo I, Leibel RL \& Ferrante AW Jr 2006 CCR2 modulates inflammatory and metabolic effects of high-fat feeding. Journal of Clinical Investigation 116 115-124. (https://doi.org/10.1172/ JCI24335)

Received in final form 10 May 2019

Accepted 28 May 2019

Accepted Preprint published online 28 May 2019 https://joe.bioscientifica.com https://doi.org/10.1530/JOE-19-0190
(C) 2019 Society for Endocrinology Published by Bioscientifica Ltd. Printed in Great Britain 